\title{
ARTICLE Programmable microfluidic genotyping of plant DNA samples for marker-assisted selection
}

\author{
Helena C. Zec ${ }^{1}$, Tony Zheng ${ }^{1}$, Lingshu Liu ${ }^{1}$, Kuangwen Hsieh ${ }^{2}$, Tushar D. Rane ${ }^{1}$, Todd Pederson ${ }^{3}$ and Tza-Huei Wang ${ }^{1,2}$
}

As demands to maintain the global food production continue to mount, multinational seed companies are turning to new DNA marker technologies to accelerate the rate of plant breeding and crop improvement. The key to widespread adoption of molecular breeding is the availability of flexible and cost-effective tools that can perform combinatorial and high-throughput genotyping of single-nucleotide polymorphisms (SNPs) to guide the crop development process. Toward this end, we have developed a programmable, droplet-based microfluidic device for genotyping maize genomic DNA. A unique feature of the microfluidic platform is the nano sample processors (NSPs), which allow the device to sequentially load an unrestricted number of unique DNA samples using only two inlets, overcoming the current limitation to the number of sample inputs due to small device footprint. Direct and programmable droplet generation within the device allows each sample to be genotyped against a panel of markers on demand. Moreover, we have successfully implemented the Invader assay for SNP genotyping in flowing, 50-nL droplets, thus achieving significant reduction in consumption of reagents per reaction as compared with conventional genotyping platforms. As a demonstration, we performed 240 Invader reactions (testing 8 DNA samples against 10 SNP markers) and achieved greater than 93\% accuracy in SNP calling of plant DNA samples in a single droplet-based experiment.

Keywords: droplets; genotyping; invader assay; microfluidics; single-nucleotide polymorphism

Microsystems \& Nanoengineering (2018) 4, 17097; doi:10.1038/micronano.2017.97; Published online: 26 March 2018

\section{INTRODUCTION}

Recent years have witnessed increasing prevalence of genomic selection technologies in the agricultural industry to accelerate plant breeding and crop improvement for maintaining stable global food supply in the face of growing human population, shrinking arable land, emerging new pests, and changing climate patterns ${ }^{1,2}$. Genomic selection relies on screening prospective crops for genetic markers that are known to confer desirable physical traits before the crops are grown, thereby fast-tracking the plant-breeding process ${ }^{3}$, while also avoiding artificial modification of crop genetics (that is, genome modification). Widespread adoption of genomic selection in plant-breeding hinges on rapid, flexible, high-throughput, and cost-effective genotyping technologies ${ }^{4}$. To this end, the agriculture industry has been focusing on high-throughput detection of single-nucleotide polymorphism (SNP) markers ${ }^{5,6}$ because of their abundance throughout the genome, stability over generations ${ }^{7,8}$, and binary-like information that simplifies genotyping and data storage ${ }^{4}$. For example, highly multiplexed nucleic-acid microarrays that rely on 'universal' sets of SNP markers ${ }^{9-11}$ have been developed for genotyping a range of plant seeds and tissues. To ensure applicability of designs across multiple species requires a large collection of SNPs, thereby increasing costs and the likelihood of ascertainment bias ${ }^{4}$. Among the genotyping systems that have been introduced to the market ${ }^{12-15}$, Array Tape ${ }^{T M}$ Technology (Douglas Scientific) is considered state-of-the-art and is used by large multinational seed companies ${ }^{4}$. This technology processes spools of Array Tape ${ }^{\mathrm{TM}}$ for assay set-up, polymerase chain reaction $(\mathrm{PCR})$, and fluorescent detection-genotyping up to 150000 data points per day. Each individual reaction is $1.6 \mu \mathrm{L}$, substantially reducing the reaction volume as compared with standard plate-based systems, which use at least $5 \mu \mathrm{L}$ reaction volumes. However, the requirement for separate and bulky modules for liquid handling, thermocycling, and detection impedes complete automation and increases costs. As such, there remains a strong interest in the agricultural industry to develop alternative genotyping technologies that are rapid, flexible, costeffective, high-throughput, and tenable for automation.

Droplet microfluidics has the potential to satisfy the unmet needs for increased miniaturization, scalability, and automation in genotyping. Using an immiscible carrier fluid (for example, oil) to discretize a single sample into thousands or even millions of individual reactions that are akin to independent benchtop reaction tubes but orders of magnitude lower in volume, droplet microfluidics drastically reduces reagent consumption and cost. Furthermore, droplets can be generated at kilohertz rates, and thus have the potential to acquire many data points in short time periods ${ }^{16}$. The utility of droplets has already been demonstrated in applications ranging from single-cell ${ }^{17-20}$ and single-molecule analysis $^{21,22}$ to particle synthesis ${ }^{23-25}$, and has also extended to genotyping ${ }^{26}$. Unfortunately, although many microfluidic droplet devices excel at generating large populations of droplets of identical composition from a single sample, a useful capability for detecting a few SNP markers from a few DNA samples ${ }^{26}$, they generally have a limited capacity for processing a large number of unique DNA samples against a panel of markers, which is crucial for high-throughput genotyping in agricultural applications. For example, microfluidic devices typically can only accommodate at

${ }^{1}$ Department of Biomedical Engineering, Johns Hopkins University, Baltimore, MD 21218, USA; ${ }^{2}$ Department of Mechanical Engineering, Johns Hopkins University, Baltimore, MD 21218, USA and ${ }^{3}$ Pioneer Hi-Bred International Inc., Johnston, IA 50131, USA

Correspondence: Tza-Hue Wang (thwang@jhu.edu)

Received: 12 July 2017; revised: 28 September 2017; accepted: 27 October 2017 
most 10-30 inlet channels due to a small device footprint, thus limiting the number of samples and reagents that can be introduced into the device for genotyping. Moreover, current microfluidic droplet devices lack versatile means of injecting sample droplets with reagents combinatorically. Several techniques to inject droplets with subsequent reagents have recently been proposed, including the picoinjector ${ }^{27,28}$ and electrocoalescence ${ }^{29}$. These devices were only able to inject a single reagent into droplets generated from a single type of sample. Such challenges in sample intake and droplet production must be addressed so that droplet microfluidics can be successfully utilized for genotyping in agricultural applications.

As a potential solution, we present herein a programmable, microfluidic platform that is capable of multiplexed genotyping of a library of DNA samples against a panel of SNP markers (Figure 1). To flexibly introduce DNA samples into the device without increasing the number of inlets and device footprint, the device features a pair of novel 'nano sample processors' (NSP) that sequentially loads multiple samples into the device via only two sample inlets (Figure 1a). When operating in unison and coupling with a robotic multiwell sampling system ${ }^{30}$, the dual NSP design can be automated to process an unrestricted number of samples for high-throughput genotyping applications without adding idle time. Within the device, each sample is digitized into droplets and each sample droplet is directly injected, without the need of droplet synchronization ${ }^{30}$, into a corresponding SNP-interrogating reagent/probe based on the isothermal and robust Invader assay chemistry (Figure 1b). As multiple DNA sample droplets are being generated and sequentially injected with reagents/probes, DNAprobe droplets further downstream are simultaneously incubated to carry out the Invader reaction and detected for an in-line fluorescence read-out (Figure 1a). Importantly, the sequence of individual droplets is maintained as it flows throughout the device, allowing for accurate droplet identification by spatial indexing. Our device therefore operates akin to a production line, eliminating the idle time encountered by typical array-based systems. This flow-based operation also lifts the constraint of the number of total reactions confined by the footprint of the device (for example, multiwell plate and arrays), thus rendering our droplet platform scalable for high-throughput genotyping. As a demonstration, we screened, in a single droplet-based experiment in our device, eight unique maize DNA samples against 10 SNPinterrogating probes in triplicates (that is, 240 Invader reactions), and achieved $>93 \%$ accuracy in SNP calling.

\section{MATERIALS AND METHODS Multiplexed PCR}

Genomic DNA extracted from eight maize samples were provided by DuPont Pioneer (Johnston, IA, USA). DNA samples were amplified using multiplexed PCR before the Invader assay ${ }^{31,32}$. The multiplexed PCR reaction contained 10 primer pairs for simultaneously amplifying 10 loci that contain the 10 target SNPs. Reagents were heated at $95^{\circ}$ $\mathrm{C}$ for $600 \mathrm{~s}$ and then thermocycled for 18 cycles: $95^{\circ} \mathrm{C}$ for $30 \mathrm{~s}, 55^{\circ} \mathrm{C}$ for $90 \mathrm{~s}$, and $72{ }^{\circ} \mathrm{C}$ for $150 \mathrm{~s}$. After thermocycling, a final extension step was executed at $72{ }^{\circ} \mathrm{C}$ for $300 \mathrm{~s}$. Before the SNP Invader assay, amplified genomic DNA targets were denatured at $100^{\circ} \mathrm{C}$ for $20 \mathrm{~min}$, snap-cooled, and then kept on ice until use.

\section{Off-chip SNP genotyping invader assay (synthetic and genomic targets)}

The Invader assay (Figure 1b) utilizes two simultaneous isothermal amplification assays in a biplex format ${ }^{33}$. The Invader assay is carried out at $63^{\circ} \mathrm{C}$ for $20 \mathrm{~min}$. In the assay design stage, five SNP markers of interest were identified and selected by DuPont Pioneer. For each SNP marker, a pair of Invader probes was designed by Third Wave Technologies to detect the two homozygous alleles and one heterozygous allele. For markers 4,

a

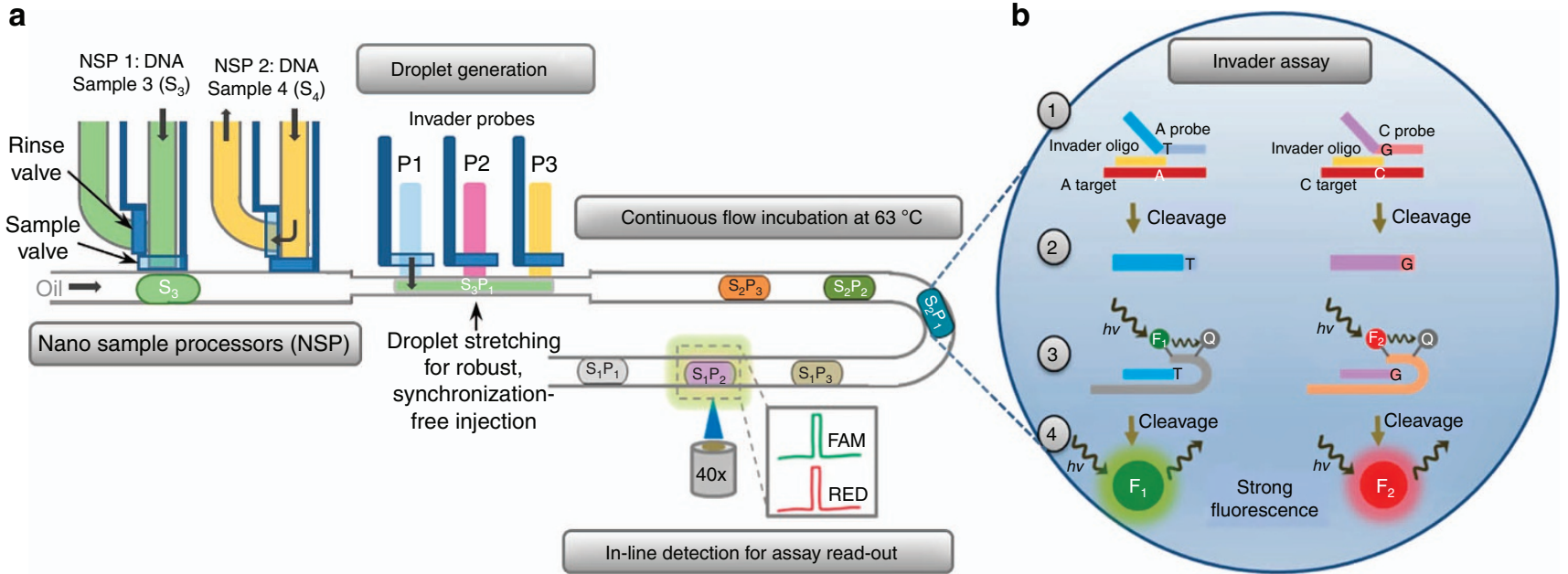

Figure 1 (a) Multiplexed genotyping of a library of maize DNA samples against a panel of SNP markers is seamlessly performed in the dropletbased platform. DNA samples are sequentially loaded into the microfluidic device via two NSPs and digitalized into $\sim 5 \mathrm{~nL}$ droplets by ondemand actuation of programmable sample valves. DNA droplets then arrive in the droplet-generation zone, where the narrow channel causes the DNA droplets to stretch and span the entire droplet-generation zone, thus allowing synchronization-free injection of $\sim 45 \mathrm{~nL}$ Invader probes and reaction mix into the DNA droplets upon actuating the corresponding probe inlet valves. DNA-probe droplets are subsequently incubated for $20 \mathrm{~min}$ at $63^{\circ} \mathrm{C}$ as they flow throughout the incubation region in a continuous flow. In-line readout is performed using a two-color fluorescence spectroscopy detection system. Fluorescence signals are then used to determine the final allelic call out for that specific DNA and probe. (b) The Invader assay consists of the target DNA, Invader oligonucleotide, flap endonuclease (FEN), and a pair of corresponding FAM (green) and RED (red) Invader signal probes and FRET cassettes. (1) In the primary reaction, both the Invader oligonucleotide and primary probe hybridize to the target of interest. An invasive structure is formed from the single-base overlap. This forms a three-dimensional structure that is recognized by the FEN cleavase, which (2) cleaves the $5^{\prime}$ arm of the primary probe. (3) In the secondary reaction, the cleaved $5^{\prime}$ arm of the probe binds to the FRET cassette. In this secondary FRET reaction, this structure is recognized by same FEN cleavase, which (4) cleaves the fluorophore and a fluorescent signal is generated. SNP, single-nucleotide polymorphism. 
$5,6,7$, and 9, synthetic, single-stranded DNA sample sets that are complementary to the probes were manufactured by Integrated DNA Technologies (Coralville, IA, USA) for initial testing. Each synthetic DNA sample set consisted of two single-stranded oligonucleotides, 'A' and 'B', one for each SNP allele. The genomic targets consisted of DNA extracted from maize seed. All genomic targets evaluated were known to be homozygous. For the genomic targets, the amplified PCR product was used in the Invader assay SNP genotyping reaction. For off-chip experimental results, concentrations identical to those being compared in the droplet format were generated in $10 \mu \mathrm{L}$ reactions in a 96-well plate. The fluorescence from the 96-well plate was then monitored on a CFX96 Real-Time PCR detection system (Bio-Rad Laboratories Inc.). Fluorescence was monitored in FAM (excitation: 450-490 nm and emission: $510-530 \mathrm{~nm}$ ) and Texas Red (excitation: 560$590 \mathrm{~nm}$ and emission: 610-650 nm). The Invader assay was carried out using corresponding Invader probes specific to the amplified targets, Cleavase XI FRET mix solution (Third Wave Technologies), Cleavase XI Enzyme solution (Third Wave Technologies), and $\mathrm{MgCl}_{2}$ solution (Third Wave Technologies). All Invader reagents' concentrations are optimized by DuPont Pioneer and proprietary.

\section{Device design}

The developed programmable microfluidic droplet device (Figure 2a) consists of two polydimethylsiloxane (PDMS) layers, utilizing a push-up valve architecture, wherein the bottom valve layer (Figure 2a, red) collapses into an upper fluidic layer (Figure 2a, green) to regulate the fluid flow. In this device, a
PDMS membrane valve is aligned underneath each fluidic inlet to enable automated control of the opening and closing of each inlet via a custom, programmable MATLAB script. The fluidic layer features a carrier oil inlet for carrier oil loading, two independent NSP inlets (Figure 2a, Supplementary Figures S1 and S2) for sample loading/rinsing, ten probe inlets (Figure 2a, P1-P10) for Invader probes/reagents, and two pressure relief channels that flank the reagent and sample inlets (Figure 2a, PR1 and PR2) for ensuring uniform droplet generation (REFs). DNA samples are digitalized into droplets $(\sim 5 \mathrm{nl})$ via the NSPs and injected with its respective Invader probe droplets $(\sim 45 \mathrm{nl})$ in the dropletgeneration zone. As DNA-probe droplets $(\sim 50 \mathrm{nl})$ flow through the device, they are mixed in the serpentine channel, incubated in the incubation zone at $63{ }^{\circ} \mathrm{C}$ for $20 \mathrm{~min}$ for the invader reaction, and detected for their fluorescence readout, before exiting via the outlet. All processes occur under flow, allowing for minimal downtime and processing of an unrestricted number of programmable droplets that allow the analysis of multiple, unique DNA samples against a panel of a flexible number of probes.

\section{Device fabrication}

Fluidic and valve layer masks were designed in L-EDIT v16.0 (Tanner EDA, Monrovia, CA, USA) and printed by CAD/Art Services Inc. (Bandon, OR, USA). Fluidic and valve layer molds were fabricated on 4-inch silicon wafers (Polishing Corporation of America, Santa Clara, CA, USA). The fluidic layer mold was patterned with SPR 220-7 (MicroChem Corp., Newton, MA, USA) to form the channel regions (height was $\sim 25 \mu \mathrm{m}$ ) to be collapsed a

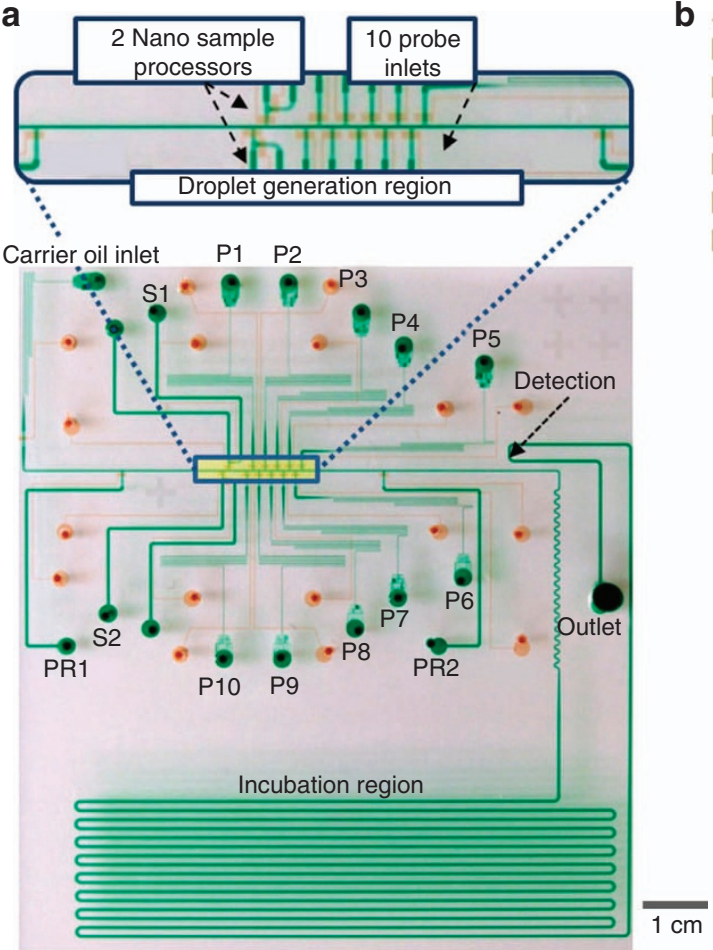

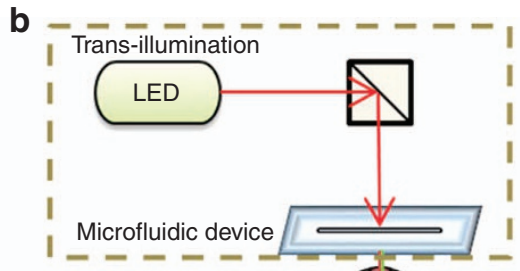

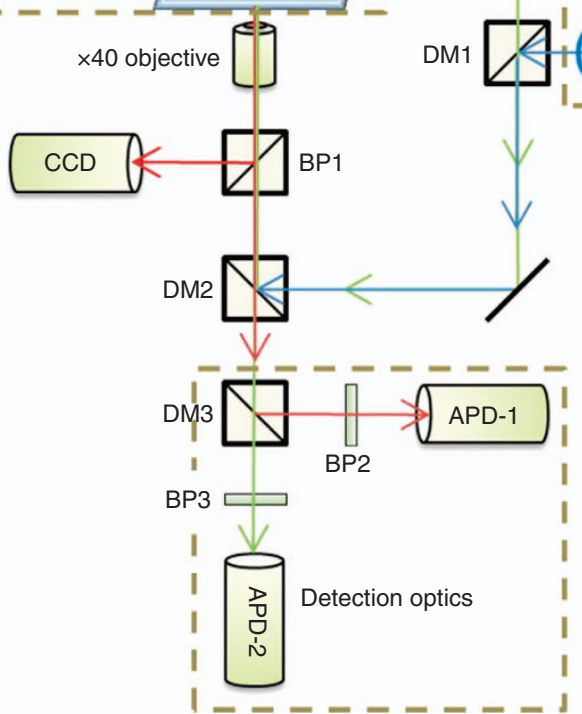

Figure 2 (a) The PDMS microfluidic device consists of two layers, a bottom valve layer (red) and a top fluidic layer (green). The fluidic layer features two nano sample processors (S1 and S2), 10 probe inlets (P1-P10), a carrier oil inlet, and two pressure relief channels (PR1 and PR2). Droplets are generated in the droplet-generation region and then travel through a serpentine channel that promotes droplet mixing. Once mixed, droplets enter the incubation region and are incubated in continuous flow at $63^{\circ} \mathrm{C}$ for $\sim 20$ min. Fluorescence read-out occurs in the detection region, near the outlet of the device using CFS. (b) The two-color CFS is designed to simultaneously detect fluorescence signals of both Invader probes within droplets. The CFS system employs two laser sources for fluorescence excitation, DM, and optical BP filters for routing optics, and two silicon APDs for detection of fluorescence signals. The instrument includes a trans-illumination LED and CCD camera for bright-field imaging of the droplets in the device. APD, avalanche photodiode; BP, band-pass; DM, dichroic mirror; CFS, confocal fluorescence spectroscopy. 
by the valve layer. This was followed by patterning of SU8-3025 (MicroChem Corp., Newton, MA, USA) to generate the rest of the fluidic channel network (height was $\sim 45 \mu \mathrm{m}$ ). For the valve layer mold, a single layer of SU8-3025 was patterned on the wafer (height was $\sim 45 \mu \mathrm{m}$ ). Widths and heights of the various channel sections in the microfluidic device are listed in Supplementary Table S1. The molds were used to fabricate the devices by soft lithography. The fluidic layer molds were first silanized using vapor deposition of chlorotrimethylsilane (Sigma-Aldrich, St. Louis, MO, USA) for $15 \mathrm{~min}$. A thick fluidic layer was then casted using $50 \mathrm{~g}$ of PDMS base to crosslinker ratio $(6: 1)$ and degassed under vacuum conditions for an hour. The fluidic layer was then cured for $8 \mathrm{~min}$ at $80^{\circ} \mathrm{C}$. The valve layer (15:1, PDMS base to crosslinker ratio) was spun at 1350 RPM (Laurell Technologies Corp., North Wales, PA, USA) and baked for $4 \mathrm{~min}$ at $80^{\circ} \mathrm{C}$. After baking, the fluidic layer was manually aligned to the valve layer under a stereoscope. Both layers were then baked for $60 \mathrm{~min}$ at $80^{\circ} \mathrm{C}$. Access holes were then punched for the inlet and outlet ports, and the PDMS device was bonded to Thickness \#1 cover glass (Ted Pella Inc., Redding, CA, USA) with oxygen plasma treatment.

\section{Device priming, hydrophobic surface treatment}

In conventional push-down valve architecture, fluidic channels reside on a hydrophilic glass surface. The bottom glass surface requires an initial hydrophobic Aquapel (PPG Industries, Pittsburgh, $P A$, USA) treatment to ensure droplet integrity and stability. Aquapel is filtered using a Syringe Durapore (PVDF) membrane filter unit, $33 \mathrm{~mm}$ diameter/0.22 $\mu \mathrm{m}$ pore size (Merck Millipore, Burlington, MA, USA), and manually injected into the device. Aquapel is flushed out with air at 10 PSI (equivalent to $68947.6 \mathrm{~Pa}$ ) followed by FC40 (3 M) at 10 PSI (equivalent to $68947.6 \mathrm{~Pa}$ ), filtered using a Syringe Nylon membrane filter unit, $30 \mathrm{~mm}$ diameter/ $0.22 \mu \mathrm{m}$ pore size (CELLTREAT Scientific Products, Pepperell, MA, USA). The device is placed in an oven at $80^{\circ} \mathrm{C}$ for $2 \mathrm{~h}$ and degassed in a vacuum. In push-up valve architecture, Aquapel treatment is not necessary and is directly degassed in a vacuum.

\section{Optimization of carrier fluid, additives, and surfactants}

Optimization of carrier oil fluid was performed for the dropletbased Invader assay. Different mixtures of fluorinated oils, including FC-40, FC-3283, HFE-7500, and $1 \mathrm{H}, 1 \mathrm{H}, 2 \mathrm{H}, 2 \mathrm{H}-$ Perfluoro-1-octanol (PFO; Sigma-Aldrich; 4:1 v/v), were evaluated. The addition of bovine serum albumin was found to improve small hydrophobic molecule and fluorophore retention within droplets, but caused droplet sticking in the channels and poor droplet mobility. These results were consistent with those found in the literature $^{34}$. A mixture of FC-40 and PFO offered the optimal balance between droplet stability and small molecule retention among the carrier oils and surfactants that were evaluated.

\section{Interface with heating}

To achieve isothermal incubation, the microfluidic device was mounted on top of a Peltier heating set-up coupled with a PID controller. The Peltier is mounted such that the incubation region of the chip lies above, and the detection region is accessible to the excitation laser while the system is kept at $63^{\circ} \mathrm{C}$ for optimal Invader assay conditions.

\section{On-chip SNP genotyping invader assay (synthetic and genomic targets)}

A set of solenoid valves was used to control the opening and closing of individual valves in the microfluidic device. The solenoid valves were controlled with a custom MATLAB (Mathworks, Natick, MA, USA) program. To interface with the microfluidic device, all reagents were loaded into Tygon ${ }^{\circledast}$ microbore tubing (Cole-Parmer, Vernon Hills, IL, USA) and connected with the device through inlet and valve ports. Valves were pressurized at 15 PSI (equivalent to $103421 \mathrm{~Pa}$ ).

The carrier oil, consisting of FC-40 (3 M) and $1 \mathrm{H}, 1 \mathrm{H}, 2 \mathrm{H}, \mathrm{PFO}$ (Sigma-Aldrich; 4:1 v/v), was pressurized at $5 \mathrm{PSI}$ (equivalent to $34473.8 \mathrm{~Pa}$ ) and loaded into the device via the carrier inlet. The carrier oil was allowed to fill and wet the entire length of the central oil channel prior to droplet generation. All reagent/probe inlets were primed and loaded with its respective reagent/probe mixture. We note that typically only $\sim 20 \mu \mathrm{L}$ of each Invader probe set was needed for a single experiment, which represents significant reduction in reagent consumption. Next, the valve actuation sequence was programmed in MATLAB (Newton, MA, USA) and executed, corresponding to the DNA-probe droplet combinations to be generated in the device. Here, the valve-opening time (typically between 0.1 and $1.5 \mathrm{~s}$ ) and the back pressure applied to the reagent/ probe inlets (typically between 1.5 and 5 PSI) were optimized to ensure the targeted droplet volumes were achieved. The digitalized DNA droplet $(\sim 5 \mathrm{~nL})$ then entered the narrowing droplet generation zone and thus stretched to span the entire droplet generation zone. A mixture containing the respective SNP-interrogating probe, enzymes, and fluorescence resonance energy transfer (FRET) cassettes $(\sim 45 \mathrm{~nL})$ was injected in a synchronization-free manner directly into the DNA droplet. Subsequent DNA-probe droplets were continuously processed in an assembly-line manner. Each DNA sample was screened against a library of 10 SNP-interrogating probes, resulting in a sequence containing 10 droplets of different probe composition per DNA sample processed. Following, each DNA-probe droplet passed through a serpentine channel region to promote mixing before entering the incubation region. The incubation region was maintained at a constant temperature of $63^{\circ} \mathrm{C}$ for Invader assay. After incubation, in-line fluorescence readout was performed on droplets using dual laser spectroscopy, where fluorescence intensity data were collected and analyzed.

\section{Fluorescence detection set-up and data analysis}

A custom-built, two-color confocal fluorescence spectroscope (CFS) is used to simultaneously detect both FAM and RED fluorescence from the Invader reactions within droplets. The CFS employs dual laser excitation (488 and $552 \mathrm{~nm}$ ) and dual emission channel (506-534 and 608-648 nm), dichroic mirrors, optical band-pass filters, and two silicon avalanche photodiodes (APD) for fluorescence detection ${ }^{35}$ (Figure 2b). The spectroscopy platform also includes a trans-illumination Light Emitting Diode (LED) and Charged-Coupled Device (CCD) camera for bright-field imaging, which is used to visually inspect and ensure that droplet generation, flow, and incubation were error-free before and during fluorescence detection.

In our platform, the APDs measure the fluorescence (in photon counts) originated from the carrier oil and the droplets as they flow through the detection region of the device and repeatedly output the total photon counts measured during a pre-set integration time (for example, $10 \mathrm{~ms}$ in this work) for the duration of the experiment. In the resulting time traces of photon counts, prolonged periods of low photon counts indicate the background fluorescence of the carrier oil and occasional, and relatively brief spikes in photon counts indicate the presence of fluorescent droplets. Detection of the droplets and measurement of their FAM and RED fluorescence intensities were achieved with custom MATLAB scripts ${ }^{35}$. Briefly, each droplet was first located based on its leading and falling edges, which were identified when the FAM fluorescence reached three standard deviations (s.d.'s) above the background fluorescence of the carrier oil. Here, because the FAM and the RED fluorescence were coincidentally excited and simultaneously detected, droplet identification via only the FAM fluorescence was sufficient. Once the droplet was identified, the average and the s.d. for both the FAM and the RED fluorescence within the droplet were calculated. In our analysis protocol, the 
average fluorescence intensity of each DNA-probe droplet (FAM, $\mathrm{RED}_{\text {DNA-Probe }}$ ) was divided by the average fluorescence intensities of the corresponding probe-only, no-target control (NTC) droplets (FAM, RED $\overline{\text { NTC }}$ ) that were performed in triplicates in the same experiment. Finally, the allelic call for the specific DNA-probe droplet was determined by the logarithmic of the ratio between the FAM fluorescence and the RED fluorescence, which we denote as $\log (\mathrm{FAM} / \mathrm{RED})$ ratio via:

$$
\log (F A M / R E D) \text { ratio }=\log \left(\frac{\frac{F A M_{\text {DNA }} \text {-Probe }}{F A M_{\overline{N T C}}}}{\frac{R_{\text {DNA }} \text {-Probe }}{R E D_{\overline{\mathrm{NTC}}}}}\right)
$$

where we had empirically determined that a ratio of greater than - 0.07 corresponds to a homozygous FAM allele, a ratio smaller than -0.39 corresponds to a homozygous RED allele, and a ratio equal to or between -0.39 and -0.07 corresponds to a heterozygous allele.

\section{RESULTS}

\section{SNP genotyping microfluidic platform}

To achieve programmable, multiplexed SNP genotyping of a library of maize DNA samples against a panel of SNP markers, we

a

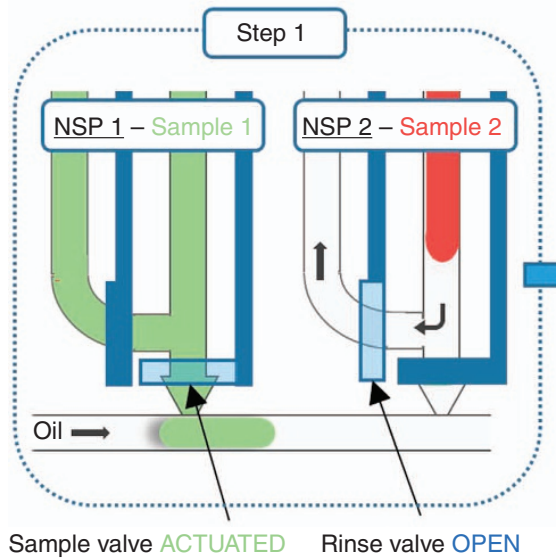

$\begin{array}{cc}\text { Sample valve ACTUATED } & \text { Rinse valve OPEN } \\ \text { for droplet generation } & \text { for sample loading }\end{array}$

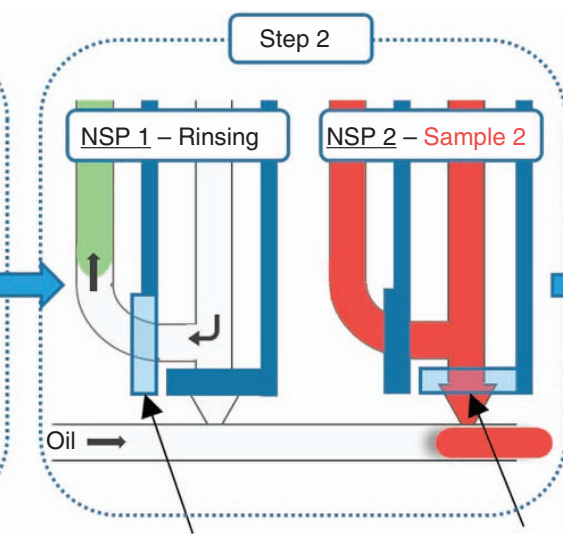

Rinse valve OPEN to flush with rinsing fluid
Sample valve ACTUATE
for droplet generation

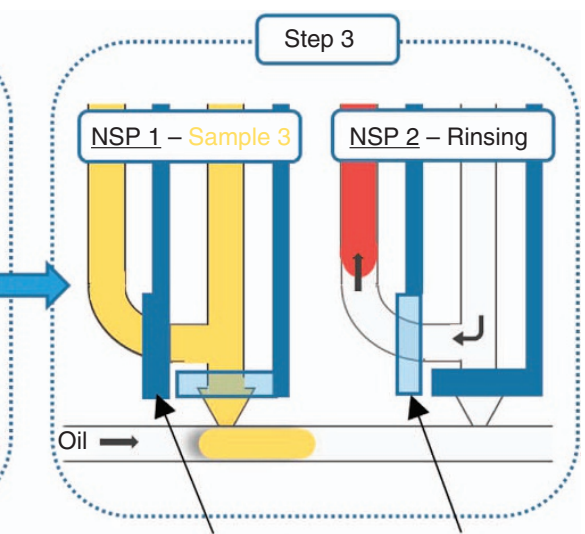

Sample valve ACTUATED Rinse valve OPEN to for droplet generation flush with rinsing fluid

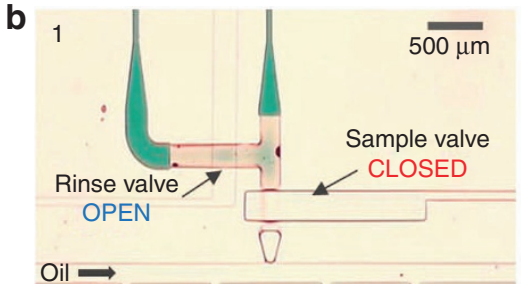

Oil $\longrightarrow$

Sample loading - Rinse valve OPEN allows rapid loading of NSP

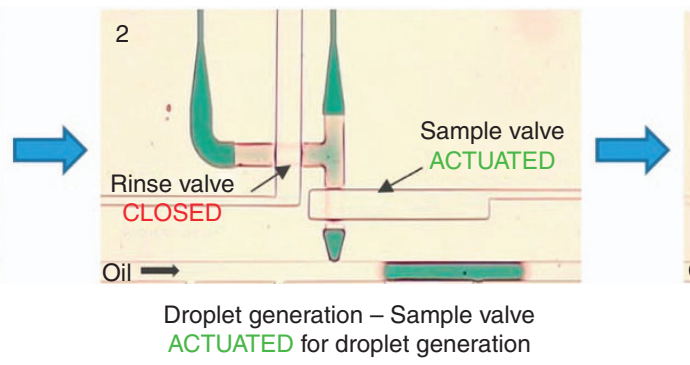

ACTUATED for droplet generation

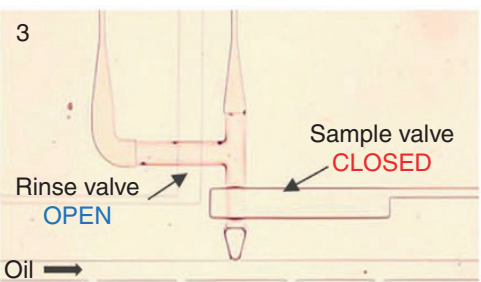

Rinsing - Rinse valve OPEN flushes sample out with rinsing fluid

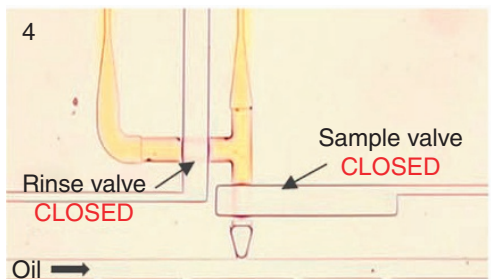

Sample switching - Rinse valve CLOSED once new sample is loaded

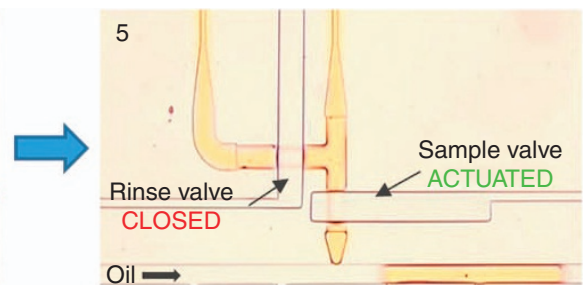

Oil

Droplet generation - Sample valve ACTUATED for droplet generation

Figure 3 (a) In the microfluidic platform, switchable dual nano sample processor inlets (NSP 1 and NSP 2) are used in tandem to process potentially unrestricted number of unique samples while minimizing downtime. Step (1) DNA Sample 1 is pressurized and loaded into NSP 1. As NSP 1 digitalizes DNA Sample 1 into a series of $\sim 5 \mathrm{~nL}$ droplets into the central oil channel, DNA Sample 2 is loaded into NSP 2. Step (2) once NSP 1 finishes digitalizing droplets from DNA Sample 1, the function of each NSP switches. NSP 2 immediately follows and digitalizes DNA sample 2 into droplets, whereas NSP 1 undergoes the rinsing step, during which the rinse valve opens and allows rinsing fluid followed by compressed air to flush and clear residual sample 1 from the NSP. Sample 3 is then loaded into NSP 1. Step (3) the function of each NSP switches back: NSP 1 digitalizes DNA sample 3 into droplets. Simultaneously, NSP 2 undergoes rinsing and loads the subsequent DNA sample. This switchable, dual NSP technology enables continuous and alternating sample processing and sample rinsing of potentially an unrestricted number of unique samples into the device via only two sample inlets with minimal idle time. (b) Step-by-step operation of NSP in processing consecutive, unique samples is visually demonstrated with green and yellow food dyes. (1) Sample loading step. By closing the sample valve and opening the rinse valve, green dye is rapidly loaded into the NSP, without flowing into the central oil channel. Once the green dye has been loaded, the rinse valve is closed. (2) Droplet generation step. The sample valve is actuated for droplet generation, digitalizing green dye into droplets into the central oil channel. (3) Rinsing step. Following droplet generation, the sample valve is closed and the rinse valve is opened. Rinsing fluid and compressed air is flown through the NSP to clean and drive the unused green dye out of the NSP. (4) Sample switching step. Following rinsing, yellow dye is loaded into the NSP and the rinse valve is closed. (5) Drop generation step. The sample valve is again actuated and yellow dye is digitalized into droplets. 
have developed a device that features a unique, high-throughput dual NSP technology, robust synchronization-free probe injection, and in-line continuous incubation and detection for assay readout (Figure 1a). DNA samples are sequentially loaded into the device and digitized into droplets via the NSP. Each NSP employs an h-shaped channel to hold fluids (for example, DNA samples and rinsing fluids) and two pneumatic microvalves to regulate their flow. The rinse valve regulates loading DNA samples into the straight segment of the $\mathrm{h}$-channel and rinsing DNA samples out of the device through the bent segment of the h-channel. The sample valve performs on-demand digitization of DNA samples in the straight segment of the h-channel into droplets into the central oil channel of the device. Moreover, we have designed dual NSPs (detailed below) such that each NSP alternates between DNA sample processing and DNA sampling loading/rinsing to maintain constant droplet generation, increase throughput, and minimize downtime. Each sample droplet moves to the downstream droplet-generation zone, and due to the narrow local channel geometry becomes elongated to overlap with all probe inlets in the droplet-generation zone, which enables direct, synchronization-free injection of a specific set of Invader probes into the droplet. The resulting DNA-probe droplet flows downstream to the incubation region on the device, which resides above a heater set at $63^{\circ} \mathrm{C}$ for performing the Invader reaction. Following incubation, droplets are sequentially detected in-line by a two-color confocal fluorescence spectroscopy system (Figure 2b). As droplets flow through the device, the sequence by which they are generated is maintained; their sequence thus provides a spatial index that precludes the need for a complicated barcoding scheme to identify each droplet. The flow-based operation within our device is similar to a production line, making our platform scalable for high-throughput genotyping.

In this work, a biplex, two-step Invader reaction is implemented within flowing droplets in our device to achieve genotyping of plant DNA samples. Here, each allele is associated with either a FAM or a RED fluorescence signal. As such, a sample with a homozygous allele is indicated by an increase in either the FAM or the RED fluorescence, whereas a sample with the heterozygous allele is indicated by increases in both the FAM and the RED fluorescence. In the primary reaction, the Invader assay uses a structure-specific flap endonuclease to cleave a three-dimensional complex formed by hybridization of allele-specific overlapping oligonucleotides to target DNA containing a SNP site for genotyping (Figure $1 \mathrm{~b})^{33}$. In the secondary reaction, the cleaved structure hybridizes to a corresponding FAM/RED FRET cassette, of which the fluorophore is cleaved by the same flap endonuclease, generating the respective fluorescence signal. Importantly, the Invader reactions in our droplets can be completed while the droplets flow through the incubation region of our device in $\sim 20 \mathrm{~min}$, and the fluorescence signals can be reliably detected by the two-color confocal fluorescence spectroscopy system to determine the specific genotype of the DNA sample within each droplet.

\section{Sample loading into microfluidic device via NSP}

Dual NSPs in our device (Figure 3a, NSP 1 and NSP 2) are designed to work in unison for loading multiple samples sequentially into the device without adding unnecessary idle time. During their operation, as NSP 1 digitalizes DNA Sample 1 into droplets into the central oil channel of the device, DNA Sample 2 is pressurized and loaded into NSP 2 (Figure 3a, Step 1). Upon completed droplet generation by NSP 1, the function of each NSP switches. NSP 2 now digitalizes DNA Sample 2 into droplets, whereas NSP 1 is first rinsed to remove Sample 1 out of the device and then loaded with incoming DNA Sample 3 (Figure 3a, Step 2). The two NSP switches role again; NSP 1 returns to digitalizing DNA Sample 3 into droplets, whereas NSP 2 now undergoes rinsing and is ready to load a new sample (Figure 3a, Step 3). As such, with each NSP alternating between sample droplet generation and sample loading/rinsing and operating in unison, a continuous workflow of sample loading, droplet generation, and rinsing is achieved without stopping the flow of the droplets in processing regions downstream in the device, thus minimizing the downtime. This dual NSP technology allows for clean and efficient loading and processing of a high number of unique DNA samples via only two sample inlets, bypassing current limitations of restricted device size and footprint that can only house 10-30 inlets. Finally, this design is even more advantageous when the dual NSP technology is interfaced with a robotic multiwell sampling system ${ }^{30}$, allowing the presented system to be operated in an automatic manner for high-throughput sampling applications.

Robust sample loading, droplet generation, rinsing, and sample switching in a single NSP was visually demonstrated using green and yellow dyes in a step-by-step manner (Figure $3 \mathrm{~b}$ and Supplementary Video S1). First, the sample valve was closed and the rinse valve was opened as the green dye was pressurized and loaded into the NSP via the sample inlet (Figure 3b, Step 1). After loading was completed, the rinse valve was closed. The sample valve was now actuated, digitalizing the green dye into droplets into the central oil channel (Figure 3b, Step 2). Of note, in our previous lines of work ${ }^{36,37}$ and current work, the size of droplets can be controlled by programming the duration of opening and closing of the sample valve. Moreover, the size uniformity of droplets is consistently $<5 \%$ in our devices, which is achieved through the addition of a pair of pressure relief channels that serve to ensure uniform droplet generation ${ }^{37,38}$. Following droplet generation, the sample valve was closed and the rinse valve was opened to allow a rinsing fluid followed by compressed air to flow through the NSP and clean any residual green dye out of the NSP (Figure 3b, Step 3). An optional rinsing fluid droplet can be generated to remove the green dye remaining in the nozzle of the NSP. After the NSP was sufficiently rinsed, a new yellow dye was loaded into the NSP before the rinse valve was closed (Figure $3 \mathrm{~b}$, Step 4). The sample valve was again actuated and yellow dye was digitalized into droplets into the central oil channel (Figure 3b, Step 5).

\section{Benchtop verification of invader assay}

The Invader assay is successfully verified with synthetic, singlestranded DNA samples on benchtop experiments. The results of the benchtop reactions served as a reference for device performance of droplet reactions and empirically establish reliable metrics for data analysis. In this work, we used five pairs of synthetic maize DNA samples (directly provided and denoted by DuPont Pioneer as sample 4, 5, 6, 7, and 9) that contain five distinct SNP markers and can be detected via their respective Invader probe sets. Each pair of DNA samples differs by only a single nucleotide and is labeled either as ' $A$ ' for the allele indicated by Redmond Red (red) fluorescence or as 'B' for the allele indicated by FAM (green) fluorescence. Here, reaction mixtures containing the reaction buffer, $\mathrm{MgCl}_{2}$, Invader probes, Cleavase, FRET cassettes, and synthetic targets were incubated at $63^{\circ} \mathrm{C}$ in a real-time PCR machine, whereas FAM and RED fluorescence signals from these reactions were measured throughout the reaction. For all five pairs of synthetic DNA samples and corresponding Invader probe sets, fluorescence intensities increased exponentially immediately after the reactions commenced and plateaued after $\sim 6 \mathrm{~min}$, indicating that the cleavage of the fluorophores from all FRET cassettes had been exhausted (Supplementary Figure S1A). The time necessary to reach steadystate fluorescence levels was incorporated into the microfluidic chip design, and determined the minimum incubation channel length. Moreover, the allele detection was specific as expected, as the ' $A$ ' alleles in all five pairs of samples resulted in an increase in 
only RED fluorescence and the 'B' alleles in all five pairs of samples resulted in an increase in only FAM fluorescence (Supplementary Figure S1B).

\section{Fluorescence detection of invader products in droplets}

Successful on-chip Invader assay in the droplet format is predicated on robust fluorescence detection of Invader reaction products within droplets. As such, we digitized Invader reaction products that were first completed on benchtop into droplets and used our two-color confocal fluorescence spectroscopy system to detect the fluorescence within the droplets. On the basis of the detected fluorescence, we first optimized the carrier oil composition that best retained fluorescence molecules within the droplet from droplet generation to detection. A mixture of $\mathrm{FC}-40$ and $1 \mathrm{H}$, $1 \mathrm{H}, 2 \mathrm{H}, \mathrm{PFO}(4: 1 \mathrm{v} / \mathrm{v})$ offered the optimal balance between droplet stability and small molecule retention among carrier oils and surfactants that were evaluated.

Using benchtop Invader reaction products digitized into droplets in our device, we also found that hydrophobic treatment of the device's fluidic channel surfaces was detrimental to the fluorescence detection of these droplets and we therefore modified our device architecture to obviate such surface treatment. Conventionally, droplet-based, microfluidic devices employ a push-down valve architecture, wherein the fluidic layer is located below the valve layer (Figure $4 a$, top). In such configuration, droplets flow directly over the bottom glass surface. Due to the hydrophilicity of the glass surface, it needs to be treated with Aquapel to render the surface hydrophobic and allow droplets to flow smoothly through the device ${ }^{18,34,35}$. However, Aquapel treatment is observed to be incompatible with the Invader assay, causing adsorption of small hydrophobic molecules, including FAM and Redmond RED fluorophores, out of the droplets and onto the PDMS channel walls. These effects further exacerbated at $63^{\circ} \mathrm{C}$. For example, when we first filled the incubation region with a number of droplets containing benchtop Invader reaction products at room temperature and then increased the temperature of the incubation region to $63^{\circ} \mathrm{C}$ as the droplets continued to flow, the fluorescence signals from successive droplets continued to decrease until the later droplets became almost undetectable (Figure $4 a$, bottom). Because these later droplets travelled through a much longer distance of Aquapel-treated channel surface, their decreased fluorescence signals strongly suggest that Aquapel inhibited fluorescence detection of Invader reaction products in our droplets. To solve this problem, a push-up valve architecture was adopted, wherein the fluidic layer is located above valve layer (Figure $4 \mathrm{~b}$, top). In this new design, droplets travel on the innately hydrophobic PDMS surface, which obviates hydrophobic surface treatment. As a result, fluorescence signals from successive droplets containing benchtop Invader reaction products were not only significantly higher than those observed with a push-down architecture, but also maintained stable signals at elevated temperatures over time (Figure $4 \mathrm{~b}$, bottom).

\section{On-chip genotyping of synthetic DNA samples}

We subsequently performed Invader reactions directly in droplets as they flowed through the $63^{\circ} \mathrm{C}$ incubation region of the device. We used synthetic, single-stranded DNA sample 6 and the corresponding Invader probe set in the initial experiment. Here, synthetic DNA sample $6 \mathrm{~A}$ was loaded through one NSP and synthetic DNA sample 6B was loaded through the other NSP, whereas the Invader probe set was loaded through a reagent inlet. We subsequently assembled multiple groups of three droplets; within each group, there were two droplets containing synthetic DNA sample $6 \mathrm{~B}$ and the Invader probe set and one droplet containing synthetic DNA sample 6A and the Invader probe set (see Supplementary Table S2 for a detailed program sequence). This asymmetric arrangement programmed by us ensured that the identities of the droplets could be readily determined from the fluorescence data trace based on which synthetic DNA sample was generated as a duplicate. After the droplets flowed through the $63^{\circ} \mathrm{C}$ incubation region in $\sim 20 \mathrm{~min}$, the FAM and RED fluorescence within droplets were measured as the droplets flowed through the detection region. In the resulting time trace showing both FAM and RED fluorescence signals, we indeed detected droplets with strong fluorescence signals, indicating that Invader reactions had successfully taken place within these droplets. Importantly, we observed repeated groups of two droplets with significantly stronger FAM fluorescence signals preceding one droplet with significantly stronger RED fluorescence signals, which matched closely with our programmed droplet sequence of two droplets containing synthetic DNA sample $6 \mathrm{~B}$ followed by one droplet containing synthetic DNA sample 6A (Figure 5a). Moreover, as multiple groups of droplets with the same sequence were observed, these results also verify a

Push-down value architecture

(Glass surface, aquapel-treated)

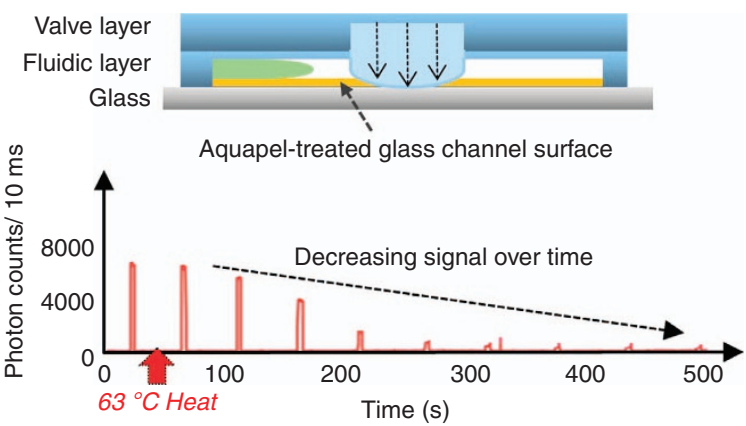

b
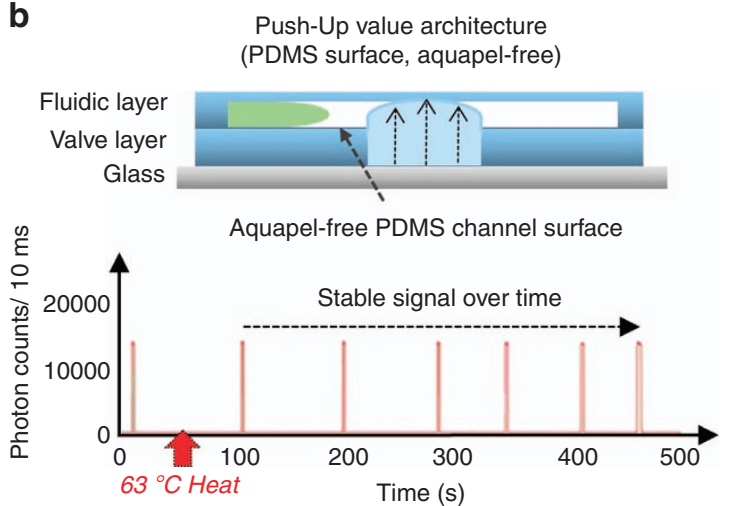

Figure 4 (a) In the push-down valve architecture, wherein the fluidic layer is located below the valve layer, droplets come into direct contact with the bottom glass surface (top). This architecture requires an initial Aquapel treatment of the bottom glass surface to ensure stable droplet mobility and integrity. Unfortunately, such Aquapel-treated device causes detrimental loss in fluorescence signals of Invader reaction products that are digitalized into droplets at $63^{\circ} \mathrm{C}$ (bottom). (b) In contrast, push-up valve architecture, wherein the fluidic layer is located above the valve layer, droplets are surrounded by PDMS (top) and can maintain stable mobility and integrity even without Aquapel treatment. By obviating Aquapel treatment, the fluorescence signals of Invader reaction products display significantly higher intensities and remain stable at $63^{\circ} \mathrm{C}$ (bottom). 

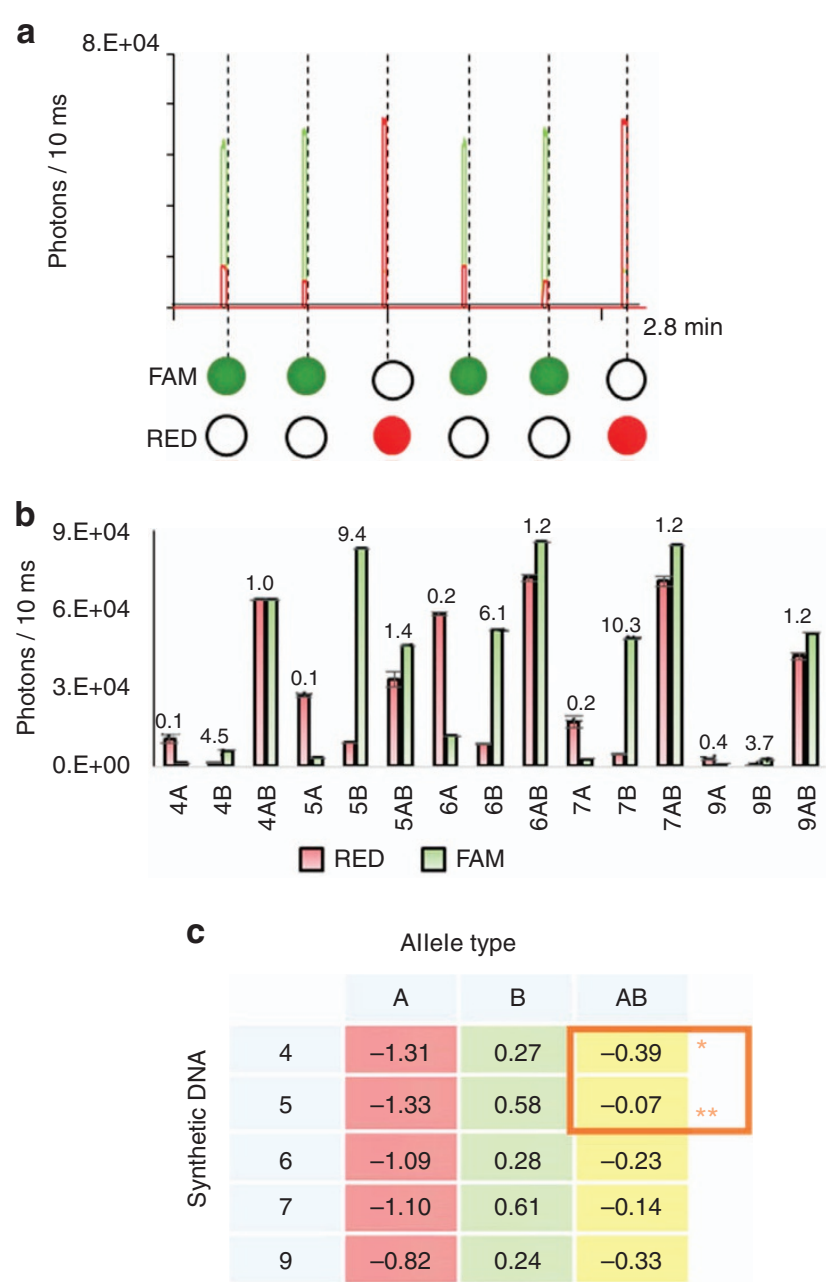

Figure 5 (a) Representative trace of synthetic target 6 FAM and RED fluorescence from on-chip, real-time read out of the Invader assay consists of two repeats of the same asymmetrical sequence, $B, B, A$. The first two peaks correspond to droplets containing synthetic target $B$ and the third corresponds to droplets containing synthetic target A. (b) The average fluorescence intensities of synthetic DNA sample sets $4,5,6,7$, and 9 with allele ' $A$ ' and allele ' $B$ ' are calculated. As expected, all ' $A$ ' alleles yielded stronger RED fluorescence, all ' $B$ ' alleles yielded stronger FAM fluorescence, and all 'AB' alleles (heterozygous) yielded comparably strong FAM and RED fluorescence. For each combination of synthetic DNA sample and SNP marker, the fluorescence intensities differed in magnitude. As a result, the ratio of FAM/RED intensity was calculated and displayed to facilitate comparison. Data shown here are prior to normalization to NTC droplets. (c) The log ratios of FAM/RED intensities of each synthetic target sets $4,5,6,7$, and 9 paired with allele ' $A$ ' and allele ' $B$ ' were calculated. Allele-calling threshold values were empirically determined from the minimum and maximum ratios of the heterozygous target (*Synthetic DNA sample 4 and ${ }^{* *}$ Synthetic DNA sample 5). Data have been normalized to NTC droplets (as shown in Equation (1)). s.d. values are shown in Supplementary Figure S3. NTC, no-target control; SNP, single-nucleotide polymorphism.

that the sequence of droplets was maintained as they moved from the droplet generation to droplet detection.

On-chip, in-droplet Invader reactions were also successfully demonstrated with $100 \%$ accuracy for all five pairs of synthetic, single-stranded DNA samples (that is, synthetic sample 4, 5, 6, 7, and 9 from DuPont Pioneer) using their respective Invader probe sets. In this experiment, each of the five pairs of synthetic DNA samples was sequentially loaded into the device, where all ' $A$ ' alleles were loaded through one NSP, all 'B' alleles were loaded through the other NSP, and each of the Invader probe sets was loaded through a designated reagent inlet. For each pair of synthetic DNA samples, multiple groups of droplets containing the ' $A$ ' allele, the ' $B$ ' allele, or an equal volume of both the ' $A$ ' and the ' $B$ ' alleles (representing the heterozygous allele) were directly injected with the corresponding Invader probe sets. As such, each pair of synthetic DNA samples generates all of the three possible alleles (that is, homozygous FAM allele, homozygous RED allele, and heterozygous allele). The NSPs were then rinsed before the next pair of synthetic DNA samples were loaded for droplet generation. After droplet incubation, droplet detection, and acquisition of the fluorescence time traces, we calculated the average fluorescence intensities for the three types of droplets from each synthetic DNA sample (Figure 5b). As expected, we measured significantly stronger RED fluorescence in droplets containing only ' $A$ ' alleles (for example, ' $4 A$ ' in Figure 5b), significantly stronger FAM fluorescence in droplets containing only ' $B$ ' alleles (for example, '4B' in Figure 5b), and comparably strong RED and FAM fluorescence in droplets containing both ' $A$ ' and ' $\mathrm{B}$ ' alleles (for example, ' $4 A B$ ' in Figure $5 \mathrm{~b}$ ). The fluorescence intensities for each combination of DNA sample and Invader probe only vary slightly, which is evident by the small error bars. Importantly, a comparison with the benchtop data shows that the on-chip Invader assay achieved a 100\% correct SNP call out for each of the 15 reactions. We note, however, that the fluorescence intensities differed significantly for different synthetic DNA samples. For example, samples 6 and 7 yielded much stronger FAM and RED fluorescence than sample 9. To facilitate efficient comparison between the synthetic DNA samples and their alleles, we therefore also calculated the FAM/RED ratio of each allele (Figure 5b; displayed above each pair of bars). On the basis of this metric, all of the 'A' alleles had ratios of $<1$, all of the 'B' alleles had ratios of $>1$, and all of the 'AB' alleles indeed had ratios of $\sim 1$.

On the basis of the results that we acquired using the synthetic DNA samples, we established a robust metric for allele calling. Here, because the fluorescence signals from different DNA samples varied significantly, a ratiometric analysis protocol was developed to determine the allele calls. Specifically, we first normalized the average FAM and RED fluorescence intensity of each DNA-probe droplet to the average FAM and RED fluorescence intensities of the corresponding probe-only, NTC droplets, which were performed in triplicates in the same experiment. Next, we calculated the logarithmic of the ratio between the normalized FAM fluorescence and the normalized RED fluorescence (Equation (1)). The $\log (\mathrm{FAM} / \mathrm{RED})$ ratios of the 15 alleles were then used to determine the thresholds for allele calling (Figure $5 \mathrm{c}$ ). As expected, the $\log (\mathrm{FAM} / \mathrm{RED})$ ratios for the ' $A$ ' alleles were negative and roughly centered around -1 , whereas the $\log (F A M / R E D)$ ratios for the ' $B$ ' alleles were positive. Notably, the log(FAM/RED) ratios of the heterozygous samples were between -0.39 and -0.07 . Consequently, we designated a ratio of greater than -0.07 as a FAM allele and a ratio smaller than -0.39 as a RED allele, and a ratio equal to or between -0.39 and -0.07 a heterozygous allele. These thresholds for allele calling that were empirically determined using synthetic DNA samples were used as the allele callout guidelines for genotyping genomic DNA samples.

\section{On-chip genotyping of genomic invader DNA targets}

On-chip multiplexed SNP genotyping of eight genomic maize DNA samples (directly provided and denoted by DuPont Pioneer as sample 1-8) against 10 Invader probe sets was successfully performed with 93\% accuracy of call out for each allele (Figure 6). Multiplexing was performed as described above and sequences of 10 droplets (one DNA sample at a time with each of the 10 probes) were generated continuously. Of the total 80 combinations, five 


\begin{tabular}{|c|c|c|c|c|c|c|c|c|c|c|}
\hline & \multicolumn{10}{|c|}{ Probe } \\
\hline & 1 & 2 & 3 & 4 & 5 & 6 & 7 & 8 & 9 & 10 \\
\hline 1 & 0.74 & 0.07 & 0.16 & 0.13 & 0.03 & 0.02 & 0.00 & 0.02 & 0.04 & 0.05 \\
\hline 2 & 0.76 & 0.69 & -1.29 & 0.63 & -0.24 & -1.58 & 0.89 & -1.18 & -1.29 & -0.96 \\
\hline 3 & -0.31 & -1.06 & -1.26 & -1.46 & -0.98 & 0.63 & 0.89 & -1.17 & -1.17 & -0.23 \\
\hline 4 & 0.70 & 0.68 & -0.01 & -1.47 & -1.47 & -1.43 & 0.89 & -0.96 & -0.96 & -0.64 \\
\hline 5 & 0.71 & 0.67 & -1.06 & -1.52 & 0.84 & -1.33 & -0.75 & -1.11 & -1.20 & -0.71 \\
\hline 6 & 0.72 & 0.67 & 0.00 & -1.60 & 0.89 & 0.73 & 0.78 & -0.20 & -1.09 & -0.63 \\
\hline 7 & -0.35 & -0.42 & 0.18 & -1.64 & -0.96 & 0.72 & 0.87 & -0.54 & -1.26 & -0.86 \\
\hline 8 & 0.69 & 0.63 & -0.94 & -1.58 & -1.37 & 0.73 & 0.88 & -1.02 & -0.03 & -0.77 \\
\hline
\end{tabular}

RED allele (ratio $<-0.39) \quad$ FAM allele (ratio $>-0.07)$

Heterogeneous allele $(-0.39 \leq$ ratio $\leq-0.07)$

Figure 6 Allele call-out table of the log of the average ratio of FAM/RED fluorescence intensities for all 80 multiplexed reactions with eight genomic Maize DNA targets against 10 Invader SNP marker probes is calculated and plotted here. Droplet sequences of 10 probes in sequential order were used for all genomic targets. Allele type is displayed in the chart as either green (FAM allele) or red (Redmond Red allele), with a log ratio less than -0.39 and ratio greater than -0.07 , respectively. Of the 80 reactions, only five resulted in a mismatched call (DNA 2 with probe 5, DNA 3 with probes 1 and 10, DNA 6 with probe 8, and DNA 7 with probes 1), resulting in a 93.8\% call accuracy. Standard deviation values are shown in Supplementary Figure S4. SNP, single-nucleotide polymorphism.

resulted in an ambiguous call when compared with the benchtop results (6.3\%). However, each of these ambiguous call outs exhibits a $\log (\mathrm{FAM} / \mathrm{RED})$ ratio in the heterozygous range, suggesting that additional data points for these particular cases may be necessary or that the assay design requires further optimization. Furthermore, the s.d.'s calculated for $n=3$ per DNA/ probe combination had low variation (Supplementary Figure S3 shows variation for synthetic targets and Supplementary Figure S4 shows variation for genomic targets), suggesting that the signals observed and consequent allelic call outs were robust. On-chip collected genotypic fingerprints for all eight maize samples matched the benchtop reactions (Supplementary Figure S1C).

Of note, accurate SNP calling from successive DNA samples indicate that cross-contamination between DNA samples was negligible in the device even when DNA samples were sequentially processed through the dual NSP inlets. Specifically, because each DNA sample had different SNP patterns for each marker studied, they were ideal samples to ensure the lack of cross-contamination. It is important to note that the Invader assay can perform up to a million-fold amplification; thus, had there been any cross-contamination between successive samples, this would have likely been observed during detection. Therefore, the rinse channel of the NSP was found to be highly effective in curtailing cross-contamination between successive genomic DNA samples, and would likely to be able to be employed in platforms that utilize other amplification-based assays, that is, the polymerase chain reaction.

\section{CONCLUSION}

This microfluidic platform represents the first embodiment of a droplet-based, continuous flow platform capable of performing the Invader assays for agricultural genotyping applications. An enabling feature of our device is the NSP, which rectifies limitations to the number of unique samples that can be introduced to the microfluidic device because of small footprint. The ability to alternate sample droplet generation between two different NSPs significantly eliminates downtime, while maintaining sample rinsing/loading and droplet processing downstream. By employing a valve-based reagent/probe fusion scheme, the microfluidic device was able to perform combinatorial screening of multiple plant DNA samples against a panel of SNP markers, where most microfluidic platforms cannot. Furthermore, the cost per screening assay is markedly lessened by reducing the reaction volume from $5 \mu \mathrm{L}$ down to $50 \mathrm{~nL}$. Importantly, we successfully implemented Invader reaction within flowing droplets that operate similar to a production line. Using our platform, we performed 240 Invader reactions (testing 8 plant genomic DNA samples against 10 SNP markers) and achieved greater than $93 \%$ accuracy in SNP calling in a single droplet-based experiment.

We envision several routes to improve our platform toward practical, high-throughput genotyping toward agricultural applications. For example, because through the current iteration of the platform we aim to demonstrate the feasibility instead of pushing for high-throughput operation, the currently reported throughput is not yet comparable to the reported throughput of traditional analysis platforms such as the Array Tape. In future iterations of the device, we can also improve the throughput by increasing the droplet generation frequency and parallelizing multiple devices. Moreover, the dual NSP design can be integrated with probe inlets, allowing probes and samples to be switched on-demand and enhancing flexibility in setting up screening reactions. When coupling the device to a robotic sampling system ${ }^{30}$, the presented system can be operated in an automatic manner for highthroughput applications. Given the current performance and the potential for improvement, we believe that our platform has the potential to fulfill the unmet need for increased miniaturization, flexibility, and scalability in genomic selection for plant breeding and crop improvement.

\section{ACKNOWLEDGEMENTS}

We are thankful to Y. Yun and H. Sullivan at Dupont Pioneer for helpful discussions. We thank the funding support from Dupont Pioneer and NSF (Grant No. 1538813).

\section{COMPETING INTERESTS}

The authors declare no conflict of interest.

\section{REFERENCES}

1 Neethirajan S, Kobayashi I, Nakajima M et al. Microfluidics for food, agriculture and biosystems industries. Lab on a Chip 2011; 11: 1574-1586.

2 Tester M, Langridge P. Breeding technologies to increase crop production in a changing world. Science 2010; 327: 818-822.

3 Rivers J, Warthmann N, Pogson BJ et al. Genomic breeding for food, environment and livelihoods. Food Security 2015; 7: 375-382.

4 Thomson MJ. High-throughput SNP genotyping to accelerate crop improvement. Plant Breeding and Biotechnology 2014; 2: 195-212.

5 Fan JB, Oliphant A, Shen R et al. Highly parallel SNP genotyping. Cold Spring Harbor Symposia on Quantitative Biology 2003; 68: 69-78.

6 Hyten DL, Song Q, Choi IY et al. High-throughput genotyping with the GoldenGate assay in the complex genome of soybean. TAG Theoretical and Applied Genetics Theoretische und Angewandte Genetik 2008; 116: 945-952.

7 Prashar S, Wolfe D, King M et al. Stability of midge tolerant varietal blends over 34 successive generations: High-speed/high-throughput, SNP-DNA fingerprinting in grain seeds. Journal of Plant Molecular Biology and Biotechnology 2012; 3: 1-10. 
8 Mammadov JA, Chen W, Ren $\mathrm{R}$ et al. Development of highly polymorphic SNP markers from the complexity reduced portion of maize [Zea mays L.] genome for use in marker-assisted breeding. TAG Theoretical and Applied Genetics Theoretische und Angewandte Genetik 2010; 121: 577-588.

9 Shen R, Fan JB, Campbell D et al. High-throughput SNP genotyping on universal bead arrays. Mutation Research 2005; 573: 70-82.

10 Steemers FJ, Chang W, Lee G et al. Whole-genome genotyping with the singlebase extension assay. Nature Methods 2006; 3: 31-33.

11 Hoffmann TJ, Kvale MN, Hesselson SE et al. Next generation genome-wide association tool: design and coverage of a high-throughput European-optimized SNP array. Genomics 2011; 98: 79-89.

12 Seeb JE, Pascal CE, Ramakrishnan R et al. SNP genotyping by the 5'-nuclease reaction: advances in high-throughput genotyping with nonmodel organisms. Methods in Molecular Biology 2009; 578: 277-292.

13 Chan M, Chan MW, Loh TW et al. Evaluation of nanofluidics technology for highthroughput SNP genotyping in a clinical setting. The Journal of Molecular Diagnostics 2011; 13: 305-312.

14 Berthier-Schaad Y, Kao WH, Coresh J et al. Reliability of high-throughput genotyping of whole genome amplified DNA in SNP genotyping studies. Electrophoresis 2007; 28: 2812-2817.

15 Magee DA, Park SD, Scraggs E et al. Technical note: High fidelity of whole-genome amplified sheep (Ovis aries) deoxyribonucleic acid using a high-density single nucleotide polymorphism array-based genotyping platform. Journal of Animal Science 2010; 88: 3183-3186.

16 Bardin D, Kendall MR, Dayton PA et al. Parallel generation of uniform fine droplets at hundreds of kilohertz in a flow-focusing module. Biomicrofluidics 2013; 7: 34112.

17 Rane TD, Zec HC, Puleo C et al. Droplet microfluidics for amplification-free genetic detection of single cells. Lab on a Chip 2012; 12: 3341-3347.

18 Brouzes E, Medkova M, Savenelli $N$ et al. Droplet microfluidic technology for single-cell high-throughput screening. Proceedings of the National Academy of Sciences of the United States of America 2009; 106: 14195-14200.

19 Mazutis L, Gilbert J, Ung WL et al. Single-cell analysis and sorting using dropletbased microfluidics. Nature Protocols 2013; 8: 870-891.

20 Klein AM, Mazutis L, Akartuna I et al. Droplet barcoding for single-cell transcriptomics applied to embryonic stem cells. Cell 2015; 161: 1187-1201.

21 Rane TD, Puleo CM, Liu KJ et al. Counting single molecules in sub-nanolitre droplets. Lab on a Chip 2010; 10: 161-164.

22 Mazutis L, Araghi AF, Miller OJ et al. Droplet-based microfluidic systems for highthroughput single DNA molecule isothermal amplification and analysis. Analytical Chemistry 2009; 81: 4813-4821.

23 Valencia PM, Farokhzad OC, Karnik R et al. Microfluidic technologies for accelerating the clinical translation of nanoparticles. Nature Nanotechnology 2012; 7: 623-629.

24 Zhang J, Coulston RJ, Jones ST et al. One-step fabrication of supramolecular microcapsules from microfluidic droplets. Science 2012; 335: 690-694.
25 Lignos I, Protesescu L, Stavrakis S et al. Facile droplet-based microfluidic synthesis of monodisperse IV-VI semiconductor nanocrystals with coupled in-line NIR fluorescence detection. Chemistry of Materials 2014; 26: 2975-2982.

26 Liu FW, Ding ST, Lin EC et al. Automated melting curve analysis in droplet microfluidics for single nucleotide polymorphisms (SNP) genotyping. RSC Advances 2017; 7: 4646-4655.

27 Rhee M, Light YK, Yilmaz S et al. Pressure stabilizer for reproducible picoinjection in droplet microfluidic systems. Lab on a Chip 2014; 14: 4533-4539.

28 Abate AR, Hung T, Mary P et al. High-throughput injection with microfluidics using picoinjectors. Proceedings of the National Academy of Sciences 2010; 107: 19163-19166.

29 Ahn K, Agresti J, Chong $\mathrm{H}$ et al. Electrocoalescence of drops synchronized by size-dependent flow in microfluidic channels. Applied Physics Letters 2006; 88: 264105.

30 Rane TD, Zec HC, Wang TH. A serial sample loading system: interfacing multiwell plates with microfluidic devices. Journal of laboratory Automation 2012; 17: 370-377.

31 Lyamichev V, Mast AL, Hall JG et al. Polymorphism identification and quantitative detection of genomic DNA by invasive cleavage of oligonucleotide probes. Nature Biotechnology 1999; 17: 292-296.

32 Lyamichev VI, Kaiser MW, Lyamicheva NE et al. Experimental and theoretical analysis of the invasive signal amplification reaction. Biochemistry 2000; 39: 9523-9532.

33 Olivier M. The Invader assay for SNP genotyping. Mutation Research 2005; 573: 103-110.

34 Courtois F, Olguin LF, Whyte $\mathrm{G}$ et al. Controlling the retention of small molecules in emulsion microdroplets for use in cell-based assays. Analytical Chemistry 2009; 81: 3008-3016.

35 Rane TD, Chen L, Zec HC et al. Microfluidic continuous flow digital loop-mediated isothermal amplification (LAMP). Lab on a Chip 2015; 15: 776-782.

36 Zec H, Rane TD, Wang TH. Microfluidic platform for on-demand generation of spatially indexed combinatorial droplets. Lab on a Chip 2012; 12: 3055-3062.

37 Hsieh K, Zec HC, Ma PC et al. Enhancing throughput of combinatorial droplet devices via droplet bifurcation, parallelized droplet fusion, and parallelized detection. Micromachines 2015; 6: 1490-1504.

38 Rane TD, Zec HC, Wang TH. A barcode-free combinatorial screening platform for matrix metalloproteinase screening. Analytical Chemistry 2015; 87: 1950-1956.

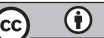

This work is licensed under a Creative Commons Attribution 4.0 International License. The images or other third party material in this article are included in the article's Creative Commons license, unless indicated otherwise in the credit line; if the material is not included under the Creative Commons license, users will need to obtain permission from the license holder to reproduce the material. To view a copy of this license, visit http://creativecommons.org/licenses/ by/4.0/

(c) The Author(s) 2018

Supplementary Information for this article can be found on the Microsystems \& Nanoengineering website (http://www.nature.com/ micronano). 